\title{
El concepto de estilema de autor en el cine: los casos de Steven Speilberg, John McTiernan y Clint Eastwood
}

\author{
David CAldeVilla DomíngueZ \\ Universidad Complutense de Madrid \\ david.caldevilla@ccinf.ucm.es \\ José RoDRÍGUEZ TERCEÑO \\ Universidad Complutense de Madrid \\ josechavalet@gmail.com \\ Juan Enrique GonZÁLVEZ VALLÉS \\ Universidad Camilo José Cela \\ juanen2012@gmail.com
}

Recibido: 29/10/2012

Aceptado: 23/01/2013

\begin{abstract}
Resumen
El concepto de 'estilema de autor' hace referencia a una marca o huella personal, identificable e intransferible, aunque sí imitable, de una manera de hacer tanto en forma como en contenido de una persona respecto a un arte. Las películas nucleares de este estudio conforman un corpus analizado que obligatoriamente sólo puede abarcar parte de la obra total, lo que se ha considerado muestra suficiente o masa crítica, para hallar en estos autores los rasgos conformantes de su personal forma de hacer. Las conclusiones se centran, a partir de estos tres autores, en los contenidos cinematográficos en los que se basa el llamado estilema autorial y su estructura.

Palabras clave: Spielberg, McTiernan, Eastwood, estilema autorial, sello cinematográfico,

\section{The Concept of Styleme of Author in Cinema: the Case of Steven Speilberg, John McTiernan and Clint Eastwood}

\begin{abstract}
The concept of 'stylemes of the author' refers to a brand or personal mark, identifiable and transferable, although imitable, of a way to do both in form and content of a person (call creator) regarding an art. Nuclear films of this study form a corpus that was analyzed and which necessarily can only cover part of the total work, which is considered sufficient sample or critical mass, to find in these authors the marks that are part of their personal way of doing. Conclusions are focused, from these three authors, in the film contents in which are based the stylemes of the author and theri and structure.
\end{abstract}

Keywords: Spielberg, McTiernan, Eastwood, stylemes, Seal film.

\section{Referencia normalizada}

CALDEVILLA DOMÍNGUEZ, David; RODRÍGUEZ TERCEÑO, José; y GONZÁLVEZ VALLÉS, Juan Enrique (2013): "El concepto de estilema de autor en el cine: los casos de Steven Speilberg, John McTiernan y Clint Eastwood". Estudios sobre el mensaje periodístico. Vol. 19, Núm. especial abril, págs.: 651-660. Madrid, Servicio de Publicaciones de la Universidad Complutense.

Sumario: 1. Introducción: el concepto de estilema. 2. Metodología. 3. Desarrollo; 3.1. Steven Spielberg; 3.2. John McTiernan; 3.3 Clint Eastwood. 4. Conclusiones. 5. Referencias bibliográficas 


\section{Introducción: el concepto de estilema}

Hace años comenzamos esta singladura con un punto de partida esencial: "En todo relato, la manera de seleccionar, agrupar, dosificar y emitir los contenidos que pretendemos transmitir es determinante. En el caso del cine la manera de narrar es tan importante que puede marcar el sentido de toda la narración o historia; es decir, el suyo específico del cine hay que buscarlo no en la historia, sino en el discurso tal y como hemos visto ya, por lo que no es aventurado afirmar que «en el cómo vemos qué se cuenta» (Caldevilla, 2005: 11). Para proseguir: "Hay dos niveles en los que se desenvuelve el relato cinematográfico: A) el lingüístico, temático o de argumento y B) el retórico, semiótico o metalingüístico. Lingüística y semiótica se unen en el cine para formar el relato cinematográfico. Esta relación será tratada de distinta forma por cada director creando así películas con su propio sello personal en el que priman los aspectos lingüísticos o los semióticos dependiendo de su forma de hacer y ser y de su concepción del cine y del relato. De su mezcla puntual y habitual por iteraciones derivaremos ya una fuente de información importante sobre la naturaleza de dicho estilema o sello personal". El fin de la gramática cinematográfica es permitir la adquisición de un «buen estilo cinematográfico», o bien de un «estilo armonioso», gracias al conocimiento de las leyes fundamentales y de unas reglas inmutables que rigen la construcción de una película. Y sobre la base del conocimiento de estas reglas, cada director o realizador podrá optar por seguirlas y adoptarlas o no, creando así su propio «efecto estilístico»". (Caldevilla, 2005: 12 y 13)

Tras varios años de trabajos analíticos textuales, seguimos conviniendo en la definición del 2005 de sello autorial (Caldevilla, 2005) entendiendo éste como la forma particular, personal e intransferible, aunque sí imitable "a la mejor manera de un falsificador de obras de arte, generalmente pictóricas", que un creador tiene de plasmar consciente o inconscientemente su aportación personal en todos y cada uno de los elementos conformantes de ese todo que llamamos obra y que como tal nos permite tomarla como objeto de nuestro estudio.

\section{Metodología}

Siguiendo el archiconocido esquema de Chatman $(1990,27)$, podemos definir el camino que toda creación ha de recorrer y, por ello, es susceptible de análisis por parte de los estudiosos del fenómeno audiovisual. En esta característica se basa la capacidad de desbrozar los constituyentes de lo que llamamos estilema autorial. Sirva el siguiente esquema de trampolín, que no de meta, para los futuros trabajos al respecto.

Por tanto el estilema autorial se ha de mostrar indefectiblemente en todos los aspectos que la narratología nos muestra y que se ven reflejados en este esquema. Bien podremos concluir que en algunos de ellos este sello no será distinguible del de otros, pero sí, por omisión -silencio- o subrayado, su conjunto, ofrecerán esta marca personal. Y para ello tomamos tres creadores estadounidenses de primera fila: Spielbreg, McTiernan y Eastwood. 


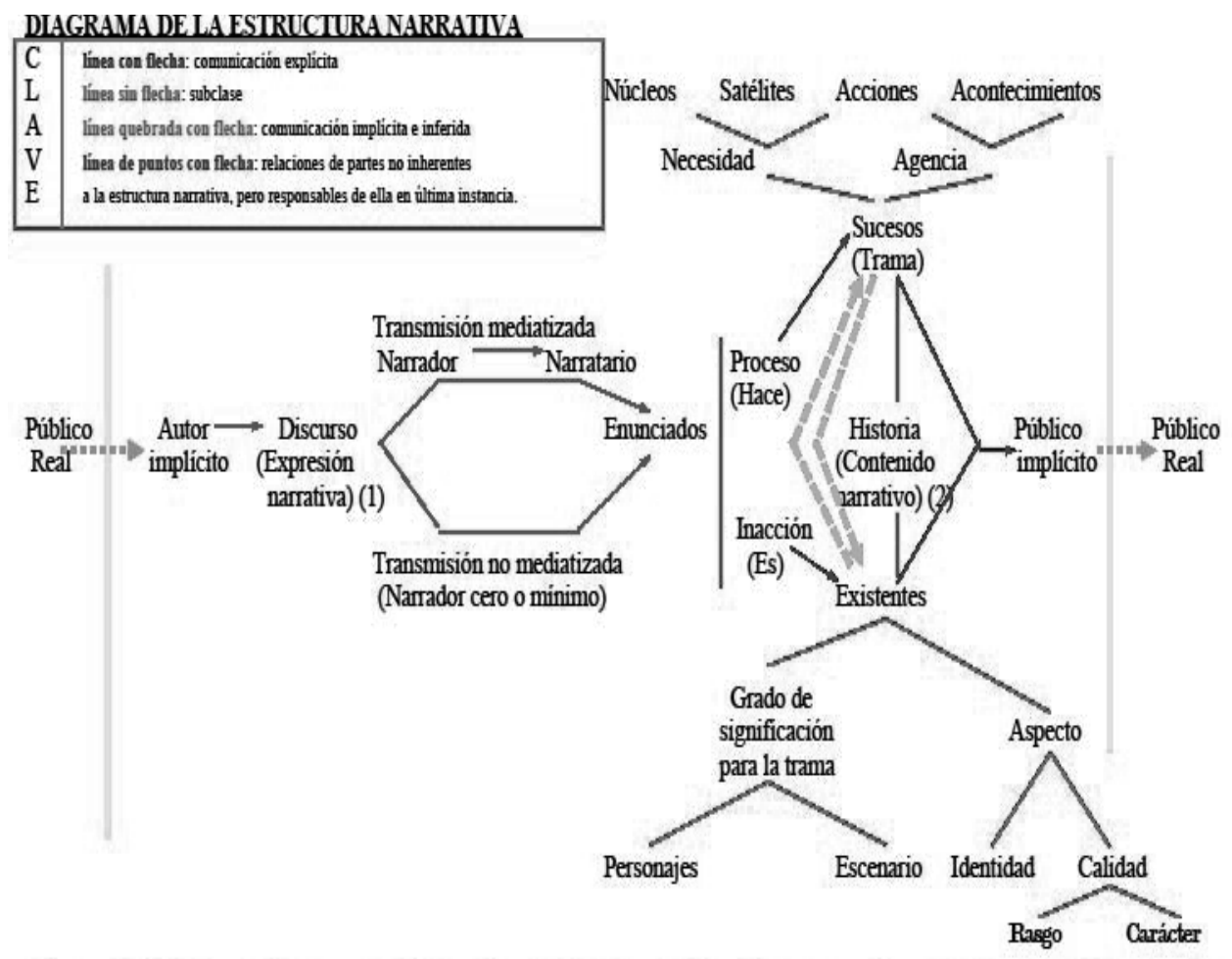

(1) Ésta es la forma de la expresión narrativa; su sustancia o manifestación aparece en varios medios (Verbales. novela, historia; Visuales: cuadros, tebeos, Audiovisuales: cine, televisión...). (2) Ésta es la forma del contenido, no la sustancia.

\section{Desarrollo}

\subsection{Spielberg}

Aplicaremos los diversos ítems del esquema anterior al sello Spielberg a partir de su tetralogía de los Indianas ${ }^{1}$.

En lo referido a la historia distinguiremos:

»Sucesos: Acciones y acontecimientos que suponen en una de las vertientes de sus obras (la comercial) una repetición de los mismos película tras película, en especial dentro de la Tetralogía de Indiana Jones creando un universo referencial de acciones y acontecimientos propios y reconocibles para el público. En la otra vertiente las acciones no son tan marcadamente propias pero sí los acontecimientos que suelen estar centrados en los aspectos de profundas convicciones del autor.

\Existentes: Personajes y escenarios. Muy reconocibles e iterados en la Tetralogía. En las demás películas de tipo comercial, los personajes son de tipo plano,

${ }^{1}$ Nos referimos a: En busca del arca perdida (1981), Indiana Jones y el Templo Maldito (1984), Indiana Jones y la última Cruzada (1989) e Indiana Jones y el Reino de la Calavera de cristal (2008). 
ya que ayudan, junto con el efecto género, a producir un ahorro importante de medios. Más que coincidencia en ellos, hay coincidencia en su trato y planteamiento. Los escenarios poseen elevadas cargas de fantasía o de realidad perfeccionada. Siempre recurre a situaciones cotidianas que se convierten en extraordinarias. En su vertiente más profunda, sus largometrajes buscan describir personajes comprometidos, que evolucionan y sienten para que empaticemos con sus causas, siempre justas y asumibles por el espectador.

» Temática, gentes, cosas, ideología y referentes: Son constantes en su Tetralogía, por motivos obvios. En su vertiente más amena, busca referentes claros, gentes reconocibles y postulados temáticos simples y poco profundos. Cuando evoluciona hacia posturas más comprometidas sus referentes culturales son los judíos o judeo-cristianos occidentales. La Esclavitud, el Holocausto, la Guerra... son lacras que la Humanidad ha sufrido y él no quiere dejar pasar por alto.

En cuanto al discurso:

» Estructura de la transmisión narrativa audiovisual: Es siempre igual en la Tetralogía, verdadero arquetipo de narración estructurada casi al milímetro, con calculados efectos en cada momento de refuerzo, atenuación, clímax, etc.. En las películas de tipo comercial recurre a los mismos elementos efectistas y en la vertiente más seria, su pulso en la dirección se hace marcadamente intenso según los momentos que crea haciendo que el tono de atención del espectador suba a voluntad. Como señala Beatriz Peña (2009: 5) "entre los puntos fuertes de Spielberg destaca el cuidado de la narrativa y el efecto emotivo sobre la audiencia".

» Manifestación: Los elementos empleados de naturaleza sonora y visual poseen una marcada tendencia a la repetición en los aspectos más importantes significativamente hablando. Empleados como refuerzo psicológico de personajes y acciones en la mayoría de las ocasiones. Spielberg emplea la iluminación, música y demás artículos que conforman el universo de la sustancia de la expresión repetidamente, como una fórmula magistral en las películas consideradas ligeras o de aventuras. En las que buscan el respeto de la Academia, su planteamiento es mucho menos efectista, cayendo a veces en una "desfiguración" de su estilema para un espectador que se mantenga en una lectura superficial, no así en la profunda pues nos ofrece varios rasgos indelebles en la amalgama del empleo de los elementos citados.

Este repaso narratológico, sirve de punto de partida para poder conjugar por activa, pasiva y omitida todos los sellos autoriales que se deseen, y con la profundidad que se requiera en cada caso ya que este esquema no se agota en su primera lectura sino que permite profundizar en cada uno de sus conformantes, llegando, por concentración de análisis hasta aspectos que podríamos tildar de atómicos dentro de esta cadena de elementos.

Por ello abrimos desde aquí estas páginas a su aplicación a casos concretos como lo son John McTiernan y Clint Eastwood, auténticos creadores, allende una conside- 
ración de buenos artesanos que siempre los acompañará dada la buena factura de sus largometrajes.

\subsection{John Mctiernan. El estilema a través del personaje protagonista}

McTiernan es un director que consigue coordinar todos los elementos intervinientes en la producción de un filme hasta amalgamarlos de una manera que refleja su particular visión del mundo, conformando eso que hemos dado en llamar sello autorial o estilo, y "si este estilo lo adorna con logros propios o que ha asimilado como propios pese a no ser de su invención, al lograr nuevos significados con ellos o nuevas combinaciones de los existentes, adquirirá el toque estilístico que marca la separación y frontera entre lo individualmente señero y lo correctamente facturado. Habrá creado su sello" (Caldevilla, 2005: 28).

Este rasgo particular en la realización y dirección del cineasta se fundamenta en colocar al individuo frente a situaciones que le superan en todos los grados posibles, siendo además unos eventos marcados por una violencia exacerbada y que van a provocar un cambio en el personaje y su relación con el medio y los demás.

Este modelo de creación, construcción y desarrollo del personaje de acción está formado por una sería de rasgos. "El Personaje, cada personaje, está construido y representado en el texto por un conjunto discontinuo de marcas, que podríamos denominar su 'etiqueta semiótica' [...] Las características de esta etiqueta, verdadero significante del personaje, vienen determinadas en buena medida por las elecciones estéticas del autor" (García Jiménez, 1993: 282).

En los aspectos físicos del personaje muestra la vulnerabilidad del cuerpo, donde el dolor, las heridas, las marcas, señalan todo el físico del individuo como consecuencia de enfrentarse a acontecimientos muy superiores a su él, caracterizadas por una despiadada brutalidad. En cuanto a sus aspectos psicológicos, el personaje aparece dotado de un alma primitiva, de una ley moral individual y de una lógica pragmática. La ley moral y la lógica de su razonamiento se verán reducidas a un estado donde el alma primitiva y violenta será la única salida posible en el enfrentamiento contra los sucesos que sufre el personaje. Por su parte, en los aspectos sociológicos la intencionalidad de la realización es mostrar como el empleo de la violencia sin una justificación de supervivencia conlleva una progresiva deshumanización del individuo y la sociedad.

Las tareas a las que se ha de enfrentar el personaje protagonista de la película de acción de McTiernan serán dos: una primera tarea que le es encomendada y que asume como una obligación; y una segunda tarea, asumida por el personaje ante la gravedad de los acontecimientos y sin que nadie se la asigne. Es un héroe a la fuerza.

La violencia que impacta sobre el protagonista, lo hace de formas diferentes y en dos tiempos: en un primer momento sufre la violencia de forma indirecta, no le atañe a él personalmente, sino a quienes le rodean; mientras que en un segundo momento la experiencia de esa violencia es directa, afectándole personalmente, atrapado en un 'tour de force' relacional. De este tipo de experiencia de la violencia y del combate final, el protagonista consigue salir victorioso superando las tareas asumidas gracias a su talento, no solo mediante el empleo de la fuerza, quedando constancia de una 
modificación en él, a nivel físico y psicológico; esa catarsis que antes mencionábamos que trasciende a su entorno aunque suele ser un paisaje deshabitado.

El espacio le es ajeno y hostil; debe adaptarse a él y que le sea útil en su objetivo. Del mismo modo, asume la limitación de tiempo para completar su empresa, respondiendo ante las situaciones de una manera rápida y concisa, produciéndose así, acciones violentas y explosivas, que marcan el trepidante ritmo de la narración. Nunca hay violencia gratuita ni el protagonista mata a nadie si no es en legítima defensa.

La base del personaje la conforman toda una serie de rasgos estereotípicos. Aunque en un primer momento los personajes pueden no parecernos 'redondos', por no contar con un elevado número de rasgos o una psicología y personalidad propia, este tipo de personajes "han sido elaborados con pocos materiales, de modo que su caracterización estática se hace fácilmente reconocible y recordable y, por ello, asociable al tipo" (García Jiménez, 1993: 304). Estos rasgos permiten una fácil identificación por parte del espectador, permitiendo una mejor lectura y utilizadas por McTiernan para crear particularidades que permiten construir un personaje que tenga una transformación, una evolución, a lo largo del relato, obteniendo así un personaje redondo por ser más humano. "Los personajes tipo no siempre han de responder a un estereotipo y, aunque lo que caracteriza no es su función, su género o su origen étnico, son sus acciones las que los definen, y están caracterizados de una forma tan general que el público los reconoce inmediatamente [...] Mientras que los personajes tipo pueden ser un elemento importante en la mayoría de historias, los estereotipos no hacen más que limitarlas" (Seger, 2000: 120); McTiernan parte de una base fácilmente reconocible por el espectador, para construir, a lo largo de desarrollo diegético y mediante la acciones y acontecimientos que se suceden, un personaje 'redondo' con una psicología y personalidad propias.

Estas características que conforman la creación, construcción y desarrollo de los personajes en la película de John McTiernan, destacan como un rasgo propio en su forma personal de hacer cine que podemos encontrar en su filmografía y un modelo de imitación por parte del cine de género de acción.

\subsection{Clint Eastwood: el sello autorial en el personaje de Harry Callahan}

El policía de San Francisco comenzó su saga en 1971 con la dirección de Don Siegel en Harry el Sucio. Película que continúa ocasionando debates sobre si su ideología es de extrema derecha o de extrema izquierda. Tanto Siegel como Eastwood contribuyeron al debate con declaraciones en varias entrevistas:

"Es sólo un hombre que se pelea contra la burocracia y una cierta forma de establecer las cosas. Emplea muchas horas en solucionar el caso y, por lo que le concierne, está más interesado en la víctima que en la ley. En Nuremberg, los americanos condenamos a gente que cometió crímenes. Los juzgamos sobre la base de que hubieran debido escuchar su conciencia en vez de seguir la ley y a sus jefes políticos. Y fue sobre esta base por la que les enviamos a la cárcel. Ocurre algo parecido con el personaje de Harry. Le dicen que las cosas funcionan así, y él contesta: 'Pues estás equivocado y yo no puede estar de acuerdo con esto'. Esto no es fascismo, es todo lo contrario al fascismo" (McGilligan, 1976, 17), sentenciaba Eastwood. 
Constantes y concesiones marcan la saga de Harry. La búsqueda del resultado será una obsesión para el inspector. Por eso, la burocracia, las triquiñuelas y las argucias administrativas van a exasperar tanto a Callahan y van a ser la causa principal de su lucha.

Los compañeros de Callahan también poseen una constante: reciben las balas que a Harry ni siquiera le rozan. Algunos mueren y otros quedan malheridos, salvo el perro que sale airoso de la película. Pero incluso en este caso el principal damnificado es Horace, el amigo negro de Callahan que le ayuda desde San Francisco y que le regala el can para que no se sienta solo en la resolución del caso en San Paulo.

En lo que ha venido a denominarse 'El Universo Callahan', Ángel Comas (2006: 151) habla de esos patrones aludiendo primero a San Francisco, lugar donde normalmente se desarrollan los hechos, pero, en este caso, sólo protagonista es los primeros minutos de la cinta, siendo San Paulo el destino de la acción a causa del destierro forzoso al que se ve sometido Callahan.

Harry recibe en todas las películas descomunales palizas y esta película no es una excepción en este sentido. Precisamente el físico también le ayuda en otro patrón, que es el de las largas persecuciones que realiza en toda la saga. En "Impacto Súbito" existen: la de los delincuentes que le lanzan cócteles molotov a su vehículo; y la carrera en su llegada a San Paulo para atrapar a un atracador.

El criminal principal de la película siempre muere a manos de Callahan, utilizando cualquier arma que esté a su disposición, y cerca del agua. Patrón que también aquí se cumple en esta cuarta entrega, puesto que Mick además tiene el privilegio de ser abatido por la Mágnum 44, precipitándose al vacío.

La forma de rodar de Eastwood es intencional en la mayoría de sus películas y aquí se acentúa incluso más. Al caer, el delincuente atraviesa el techo de un tiovivo. Objeto que forma parte de la pesadilla que desde hace años acompaña a la propia Spenser, al ser violada junto a su hermana muy cerca de ese lugar. La acción va a encaminarse hasta allí cuando Spenser consigue escaparse de Mick, y en el que juega al gato y al ratón para ganar tiempo.

Justo en medio de ese juego, Mick pasa por delante de un unicornio y el plano se queda fijo en el cuerno del animal mitológico, estableciendo, presuntamente, una conexión entre la muerte del criminal y ese elemento. Sin embargo las escenas transcurren y, cuando ya parece totalmente olvidado, Mick atraviesa el techo del tiovivo y va a incrustarse precisamente en el cuerno olvidado del animal mitológico ${ }^{2}$. Eastwood demuestra que su experimentación detrás de la cámara es ya suficiente y empieza a dominar a su antojo los tiempos y la narrativa.

Establecemos aquí otro que no sólo es patente en toda la saga, sino en toda la filmografía de Eastwood. Las secuencias de apertura y cierre se ruedan todas de forma aérea.

2 El tiovivo de hecho recuerda al final de "Extraños en un Tren" (1951) de Alfred Hitchcock, mientras que el unicornio lo hace a la entonces reciente "Blade Runner" (1982) de Ridley Scott. 
Impacto Súbito es un proyecto que llevará la firma Eastwood en contenido y forma, donde ya respeta sus postulados iniciales, provinientes de películas como Primavera en Otoño (Gonzálvez, 2009: 4). La oscuridad empieza a ser huella imborrable en las cintas del de Carmel. Entroncamos el uso de la fotografía con el del tratamiento de los personajes. Si las sombras de la pintora se maximizan, no va a ser menos el tratamiento que se le otorgue a Callahan porque los personajes están claramente en relación.

Eastwood otorga la información sobre la autoría de los crímenes al espectador desde el primer momento. El californiano no se cuestiona el quién sino el porqué de los hechos y, sobre todo, vuelve a mostrar su teoría sobre el espectador inteligente, al que él se dirige, contradiciendo a Dwight MacDonald, "al que Umberto Eco califica de apocalíptico en el sentido de que éste plantea un enfoque pesimista acerca de la influencia de los medios globales de comunicación" (Torres Hortelano, 2011: 54)

Concluimos entonces remarcando las huellas que Eastwood deja en esta película. Estilemas que son propios de su sello autorial y que se introducen en esta saga sin perjuicio de la misma. Más bien al contrario, Eastwood construye, desde las constantes de la saga de Callahan, la película más sobresaliente de la misma, al poner sus dedos (metafóricos, claro está) sobre el proceso de construcción de esta obra cinematográfica.

\section{Conclusiones}

Para establecer las conclusiones oportunas, acudiremos de nuevo al esquema propuesto por Chatman. En cuanto a la historia establecemos:

» Sucesos: Al tratarse de tres sagas cinematográficas, Spielberg, McTiernan y Eastwood condicionan el universo de las acciones al de los personajes. Es decir, todos los acontecimientos que acontecen en las películas analizadas son propios de los personajes protagonistas y los espectadores los reconocen como tal.

»Existentes: (personajes y escenarios) Que dominan las sagas de Indiana, McClane y Callahan. Son personajes sin demasiadas aristas y con defectos y virtudes muy reconocibles para todos sus seguidores. Especialmente relevante resulta el estudio del personaje en el cine de McTiernan, desarrollando en profundidad la psicología interior (la propia del personaje) y exterior (la que le atribuye el autor).

Los escenarios de los personajes de este trío de directores siempre van a tener una constante, la de que sucedan cosas difíciles de observar en el quehacer cotidiano.

» Temática, gentes, cosas, ideología y referentes: Spielberg, McTiernan y Eastwood también ponen en común la búsqueda de referentes cristalinos, que resulten permeables para el público que los observa.

En referencia al discurso:

» Estructura de la transmisión narrativa audiovisual: El esquema de los directores analizados pone el acento en la acumulación de intensidad que desemboque en el clímax y posterior desenlace de la película. Es un esquema habitual en el cine comercial que, quizá, en los casos de sagas cinematográficas se acentúe todavía un poco más. 
» Manifestación: Destaca el uso de la iluminación, y su consecuencia sobre la fotografía, que realizan estos tres directores. Establecemos un empleo psicológico de la misma que ayude a descodificar la naturaleza de los intervinientes en una acción, es decir, tanto personajes como escenarios.

\section{Referencias bibliográficas}

ALTMAN, Rick (2000): Los géneros cinematográficos. Barcelona, Paidós Comunicación.

CALDEVILLA DOMÍNGUEZ, David (2005): El sello de Spielberg. Madrid, Visión Net.

CASAS, Quim (2003): Clint Eastwood. Avatares del último cineasta clásico. Madrid, Jaguar.

CHATMAN, Seymour (1990): Historia y discurso. Madrid, Taurus Humanidades.

COMAS, Ángel (2006): Clint Eastwood: Tras las huellas de Harry. Madrid, T\&B.

FOOT, John H. (2009: Clint Eastwood: evolution of a filmmaker. Westport, Praeger.

GARCÍA JIMÉNEZ, Jesús (1993): Narrativa audiovisual. Madrid, Cátedra.

GONZÁLVEZ, Juan Enrique (2009): "Primavera en Otoño de Clint Eastwood" en Revista de Comunicación Vivat Academia, $\mathrm{n}^{\circ}$ 109. Madrid. Universidad Complutense: http://www.seeci.net/vivataca/numeros/n109/Num109/PDFs/n109-Cine2.pdf [fecha de consulta: 12 de agosto de 2012]

MAURER, Isabel (2011): “Cruzando fronteras: Todo sobre mi madre (1999) de Pedro Almodóvar" en Revista de Comunicación SEECI, $\mathrm{n}^{\circ} 24$. Madrid. Universidad Complutense: http://www.ucm.es/info/seeci/Numeros/Numero 24/DATOS.html [fecha de consulta: 11 de agosto de 2012]

MITRY, Jean (1989): Estética y psicología del cine. Madrid, Siglo XXI.

PEÑA, Beatriz (2009): "La emoción en el cine" en Revista de Comunicación Vivat Academia, $\mathrm{n}^{\circ}$ 102. Madrid. Universidad Complutense: http://www.ucm.es/info/vivataca/anteriores/n102/DATOSS102.htm [fecha de consulta: 30 de agosto de 2012]

SEGER, Linda (2000): Cómo crear personajes inolvidables. Barcelona, Paidós Comunicación.

TORRES-HORTELANO, Lorenzo J. (2011): "De lo vernacular y el World Cinema en Biutiful" en Revista de Comunicación SEECI, $\mathrm{n}^{\circ}$ 24. Madrid. Universidad Complutense: http://www.ucm.es/info/seeci/Numeros/Numero 24/DATOS.html [fecha de consulta: 1 de agosto de 2012] 


\section{David CALDEVILLA DOMÍNGUEZ}

Universidad Complutense de Madrid.

Facultad de Ciencias de la Información

Profesor del departamento de Comunicación Audiovisual y Publicidad II david.caldevilla@ccinf.ucm.es

\section{José RODRÍGUEZ TERCEÑO}

Doctorando en Comunicación Audiovisual por la Universidad Complutense de Madrid. josechavalet@gmail.com

\section{Juan Enrique GONZÁLVEZ VALLÉS}

Universidad Camilo José Cela

Profesor e investigador

juanen2012@gmail.com 